\author{
Hypertension Curriculum Review \\ Donald G. Vidt, MD, Section Editor
}

\title{
Genetics and Hypertension
}

\author{
Alan B. Weder, MD
}

\begin{abstract}
Although it has long been appreciated that there is a genetic component to hypertension, it is only recently that specific genes are being identified. This progress is part of a general advancement of our understanding of common, genetically complex human diseases. This understanding will eventually affect clinical practice. (J Clin Hypertens. 2007;9:217-223) ${ }^{\circledR} 2007$ Le Jacq
\end{abstract}

G enetic variants contributing to human hyper-

Itension have been preserved by natural selection (and the process of genetic drift), but it is highly unlikely that in an evolutionary context, there are "genes for hypertension." High blood pressure (BP) itself is not associated with any obvious impairment of reproductive success because it exerts its deleterious cardiovascular and renal effects almost exclusively during the postreproductive years where its genetic underpinnings should be largely invisible to the force of natural selection. Thus hypertension is likely to be a byproduct of genetic variants preserved for other functions, and it is identification of those functions that may yield insights into the genes contributing to high BP.

Since the functions contributing to hypertension are complex, it is not surprising that essential hypertension is also genetically complex (ie, there are many genes interacting in unpredictable ways). Currently, even the general nature of gene-gene and protein-protein interactions as they relate to

From the Division of Cardiovascular Medicine, Department of Internal Medicine, University of Michigan, Ann Arbor, MI

Address for correspondence:

Alan B. Weder, MD, 24 Frank Lloyd Wright Drive,

Lobby M, Ann Arbor, MI 48106

E-mail:aweder@umich.edu

ID: 6587 polygenetic diseases is poorly understood, and there is an understandable desire to reduce the complexity of the problem by limiting attention to single gene variants. Finding individual variants with a measurable effect on BP has proven difficult, as one would expect in a genetically complex disease, but as we uncover variants in more and more genes, we may be able to understand how those genes and proteins cooperate in the biologic pathways that produce hypertension.

In addition to complexity at a genetic level, hypertension is a physiologically complex disease because $\mathrm{BP}$ regulation is the product of a hierarchically organized, highly integrated cardiovascular system. Hypertension cannot be characterized as a disorder of any single part of that system; it is an emergent property of the whole. Currently, studies of hypertension are dominated by reductionist approaches that are often aimed at isolating biochemical or cellular signaling mechanisms thought to promote hypertension. Such approaches frequently ignore the interactive effects of those mechanisms at higher biologic levels, where emergent properties dominate function. The proper methods for the study of human hypertension therefore belong to the discipline of systems biology.

Another way some have sought to simplify gene discovery has been to seek answers in animal models of hypertension. While animal models have been of undoubted importance in understanding the biochemistry and physiology of hypertension, they are unlikely to be important in gene discovery, because while inbred hypertensive strains mimic essential hypertension, they fail as true models (ie, simplified representations of more complex systems). All hypertensive animal strains have been created by indirect (inbreeding with truncating selection for BP level) or direct (knockout, overexpression) gene manipulations that are targeted to produce animals with high BP. While it is remotely 
possible that such modeling could artificially select the same genes that have been naturally selected in humans over the ages, the premise seems a priori untenable. Even though genetic studies are more difficult in humans than in animals, we are well advised to focus on man when searching for the genetic determinants of hypertension.

Finally, hypertension results from the interaction of genes and environment. While it is commonly stated that some $40 \%$ of hypertension is attributable to genes, it is actually impossible to separate discrete genetic and environmental components. Consider, for example, a situation in which all environmental factors are to be held completely constant; any residual variation in BP would be genetic. On the other hand, if genes were identical, variation would be completely environmentally determined. In the real world, both genes and the environment contribute. Thus, whatever the genetic variants associated with hypertension are, they result in high BP only when they interact with permissive environmental factors. It is also clear that with rare exceptions, genes are not highly deterministic (ie, they do not always produce the same hypertensive phenotype in different environments). Thus, when we speak about genes for hypertension, we must also consider the environment in which those genes operate.

\section{NATURAL SELECTION AND HYPERTENSION}

Since hypertension is not a direct product of natural selection it must be a byproduct of genes selected for something else. Most hypertension is found in association with other diseases of civilization, including obesity, diabetes mellitus, and dyslipidemia; the traits of those diseases are also genetically associated. It is therefore likely that hypertension is 1 facet of a set of genes preserved to deal with problems commonly encountered by our Paleolithic ancestors, where the unpredictability of the availability of nutrients was life threatening. These genetic complements are now rendered harmful by our present environment of excess.

Body mass index is a correlate of BP, and obesity in youth is a strong predictor of adult hypertension. Similar observations relating body fat to diabetes mellitus prompted the late James Neel to propose the "thrifty genotype" hypothesis, which posited an ancient selective advantage that preserved genes capable of promoting fat storage during times of surfeit and enhanced survival during times of want. While well adapted to our Paleolithic ancestral environment, in modern societies such genotypes promote obesity. Several studies have identified genomic segments that apparently harbor genes linked to obesity, and it is likely that some of those genes will also be associated with hypertension.

Hypertension and diabetes are frequently found together, and even nondiabetic individuals with hypertension often demonstrate impaired fasting glucose, postprandial glucose intolerance, or insulin resistance. These insulin-glucose abnormalities may represent developmental stages of a metabolic syndrome that is expressed most dramatically in the presence of obesity. Some have argued that insulin resistance causally underlies the predisposition to hypertension, but at this time, the demonstrated relationship is limited to an association.

Dyslipidemia is closely associated with insulin resistance in both obese and nonobese individuals and is frequently associated with hypertension. High triglyceride and low high-density lipoprotein cholesterol levels cluster with hypertension in families, particularly in obese individuals within those families. Some $12 \%$ to $16 \%$ of the hypertensive population may have dyslipidemic hypertension, and the underlying genetic predisposition is clearly strongly aggravated by modern environmental factors promoting obesity.

The aggregation of hypertension, obesity, disordered insulin-glucose metabolism, and dyslipidemia suggests that they are all phenotypic aspects of a syndrome resulting from the expression of a set of genes originally preserved for enhancing the storage of calories and other nutrients, including sodium, which have now become problematic when expressed in our modern environment.

\section{WHAT IS THE NATURE OF THE GENES INVOLVED IN HYPERTENSION?}

$\mathrm{BP}$ is a quantitative trait with continuous variation from low to high values, with no obvious break point in the population distribution. Such quantitative traits are usually polygenic (the product of many genes). Indeed, when the heritability of hypertension was established in comparisons of BP values in identical and nonidentical twins, transmission patterns of BP in extended families and cross-sectional measurements of $\mathrm{BP}$ in adoptees and biologic children within families were most consistent with polygenic inheritance. Estimates of the contribution of genetic factors derived from such studies suggest that somewhat less than half of the populational variation in BP can be attributed to genes; however, as noted above, partitioning phenotypic variation into genetic and environmental components can be performed only in a particular set of environmental circumstances, as all hypertension is the result of gene-environment interactions. 
Genes contribute to hypertension if they have allelic variants that result in increased (or decreased) BP. The standard model holds that the effects of these multiple alleles are additive, each genetic variant contributing a small increment or decrement to the BP level. The situation is undoubtedly more complex, with interactions at the genomic, gene expression, posttranslational (alternative splicing), and protein levels. Even after these factors are explicated, as noted above, hypertension results only when genetic differences affect the integrated functions of cardiovascular and renal BP regulation.

\section{GENE-ENVIRONMENT INTERACTIONS}

Hypertension cannot be understood without appreciating the critical role of gene-environment interactions. The function of the environment is permissive, as evidenced by cross-cultural population studies. George Rose first pointed out that when a disease is defined by an arbitrary threshold value (eg, $140 \mathrm{~mm} \mathrm{Hg}$ systolic BP for hypertension), the prevalence of hypertension is a function of the population mean, with perhaps an additional contribution of skewing of the population distribution. Populations comprise individuals with differing genetic complements, and when a population of individuals is exposed to a common set of environmental influences, some respond more vigorously than others based on how their sets of genes interact with the environment. This is known as a norm of reaction. In large populations, if most individuals have at least some response, the entire population distribution shifts to a higher level. If only some individuals respond, the distribution spreads and becomes skewed. In either case, universal exposures result in an increase in the proportion of individuals with hypertension. Measurement of ecologic differences in the environment of populations of differing prevalences of hypertension can suggest causal factors; the best-studied ecologic factors associated with hypertension are dietary sodium intake and obesity. Environmental factors can also be suggested by intrapopulational studies of exposures of individuals to factors that track with the phenotype of interest; alcohol use is an example for hypertension.

In all cases, however, factors identified either in cross-population or intrapopulation association studies do not prove causation. Therefore, experimental studies testing the effects of manipulations of a proposed casual factor (eg, sodium) are required to support a causal role. This is the purpose of clinical trials in which groups of individuals with similar initial BP phenotypes receive different exposures to the element of interest: differences in mean responses support a causal role for the factor in promoting hypertension. In addition, the heterogeneity of the responses of individuals to common exposures can also tell us something about the underlying genetic determinants of the response. Differential responsiveness of BP to manipulation of sodium intake is a well-studied example. Such differences may reasonably be presumed to result from an interaction of dietary sodium with the unique set of genes of an individual, and therefore genetic studies identifying sets of alleles differing between sodium-sensitive and sodium-resistant hypertensives might reveal which variants are associated with responsiveness.

Young and colleagues have recently presented fascinating new evidence of gene-environment interactions in their observations of latitudinal clines for allelic variations of genes encoding proteins central to sodium handling. Latitude has been shown in the past to be a strong selective force shaping body habitus, probably to optimize the body's handling of heat dissipation. Because sweating is a major source of electrolyte loss, genetic mechanisms promoting sodium retention would be favored in hot climates, and the authors hypothesize that genes adapted to hot, arid environments could promote hypertension in our present milieu. The GNB3 ( $\mathrm{G}$ protein $\beta-3$ subunit) $825 T$ allele shows a particularly striking latitudinal gradient, and using data from 35 populations in the International Study of Sodium, Potassium, and Blood Pressure (INTERSALT) and genotypes from the Human Genome Diversity Project, a very strong association of the GNB3 $825 T$ allele with hypertension was observed. Thus GNB3 is a likely gene for which natural selection of an allele favoring sodium conservation and vasoconstriction in our ancestors now promotes hypertension susceptibility in the modern environment. Since GBN3 $825 T$ is only one of several genes known to have heat-adapted alleles, the impact of progressively colder environments with migration away from the equator may be one of the most important forces shaping a genetic predisposition to hypertension.

Finally, there appear to be aspects of intrauterine development that condition a fetus to develop a phenotype that predisposes to adult hypertension and the other elements of the metabolic syndrome. The idea is based on observations of an inverse relationship of birth weight and future risk of cardiovascular disease. The relationship is hypothesized to result from a "predictive adaptive 


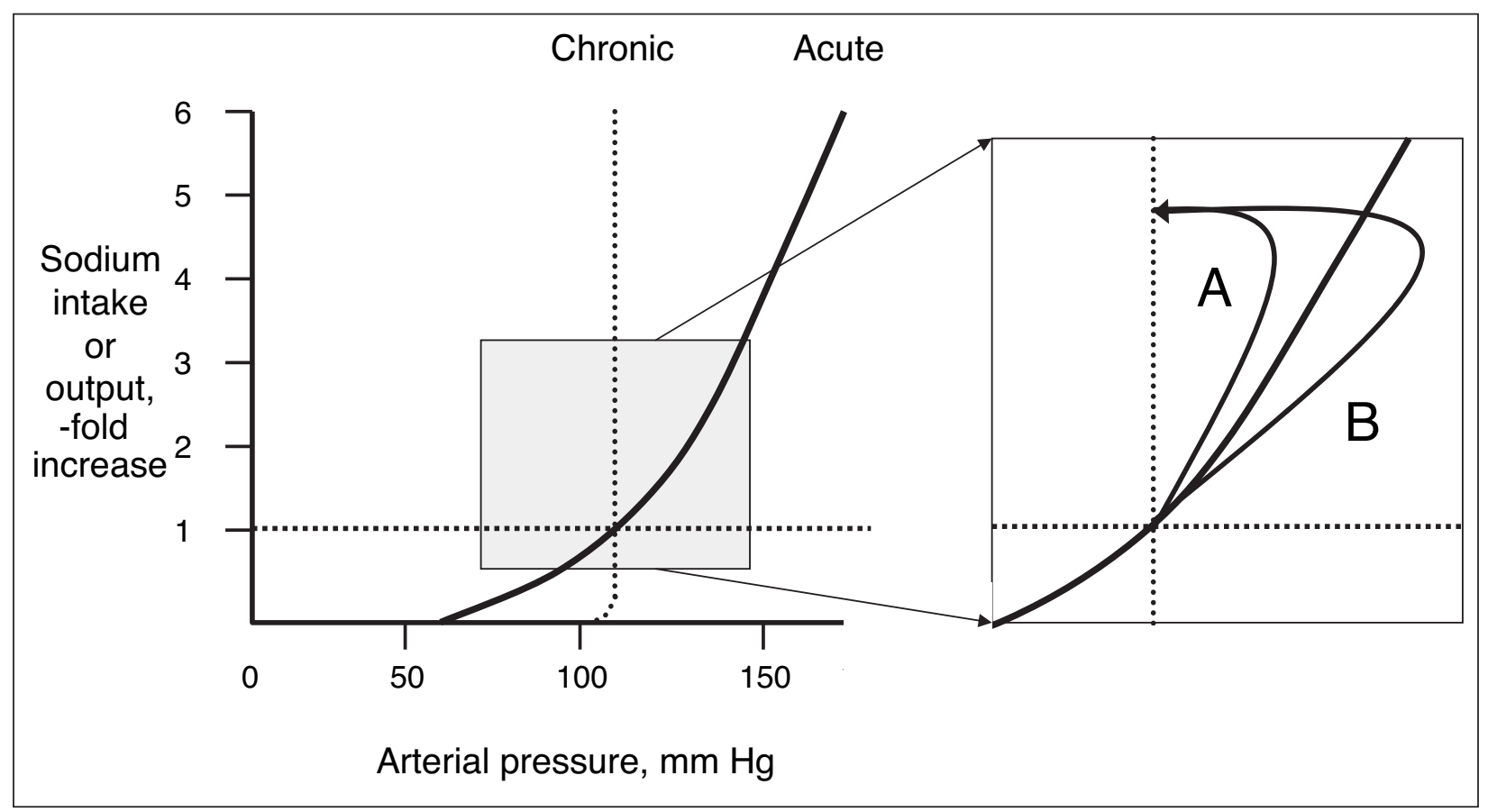

Figure. The acute and chronic relationship of sodium excretion and blood pressure. During normal sodium intake (horizontal dashed line, arbitrary value of 1), the intersection of the chronic pressure-natriuresis curve (vertical dashed line) and the sodium intake line defines the set point of blood pressure. The solid line (acute) describes the short-term blood pressure response to an abrupt increase in sodium intake. As shown in the insert, blood pressure rises transiently, but over the course of days returns to the chronic pressure-natriuresis line without a change in the set point for blood pressure regulation despite the continuation of the higher sodium intake. Two different trajectories of the restoration of chronic sodium balance are depicted. Curve A represents an individual in whom sodium balance is restored with little change in blood pressure (sodium resistant); the excursion of blood pressure is greater in the individual represented by curve $B$ (sodium sensitive).

response" in which the fetus responds to cues from the environment by adjusting its metabolic state to deal most efficiently with the anticipated postnatal setting while still within the intrauterine environment. If the metabolic programming adjusts the phenotype to deal with an environment of scarcity but the actual environment proves to be calorierich, the intrauterine adjustments are inappropriate and result in excessive fat accumulation and, by adulthood, the development of the metabolic syndrome. This ability of the fetus to shape itself to a "thrifty" profile represents yet another way genes and environment interact to produce phenotypes.

\section{HOW GENETIC VARIATION CAUSES HYPERTENSION}

The cardiovascular system is complex and integrated; BP is an emergent effect of the system as a whole. Using a variety of experimental models, Guyton and his colleagues developed a model of circulatory physiology that describes the relationship of sodium and water intake to BP regulation. While there are numerous mechanisms that transiently affect BP in this model, long-term volume and $\mathrm{BP}$ homeostasis are achieved by pressure natriuresis and diuresis effected by the kidney (Figure). Pressure natriuresis is a classic negative feedback system. When arterial BP rises, renal excretion of sodium and water ensues and continues to do so until the $\mathrm{BP}$ is restored to a point at which volume intake and output are matched. Thus, without an alteration in the set point of renal pressure natriuresis, there can be no sustained increase in BP.

There are a number of ways to change the pressure-natriuresis set point, the most obvious of which is a change in kidney function (ie, a decrease in glomerular filtration rate). Lower glomerular filtration rate resulting from either disease or normal senescence with aging compromises the ability of the kidney to excrete normal amounts of sodium, and the system adjusts by operating at a higher set point of BP. Essential hypertension, however, is not associated with any obvious decline in glomerular filtration rate, suggesting that other mechanisms must raise the set point of BP. Based on the observation that the rare monogenic forms of hypertension and hypotension result from disturbances of renal sodium handling caused by mutations of the genes encoding proteins involved in renal tubular 
sodium transport mechanisms, it seems plausible to suggest that subtler disorders of these transporters or their regulatory mechanisms could underlie the diathesis to essential hypertension.

In addition to the mutations that cause monogenic forms of hypertension, there are a number of other genes with alleles related to hypertension (Table). A number of them are associated with the salt sensitivity of BP. The example of GNB3 has been described above. A sodium-retaining allele affecting the promoter of the angiotensinogen $(A G T)$ gene shows a similar latitudinal cline. In the case of $A G T$, the mechanism of sodium retention appears to result from increased expression of the gene in the kidney, which raises local angiotensin II levels and stimulates renal tubular sodium reabsorption. Cytochrome P450 3A5, an enzyme that metabolizes a wide variety of endogenous substrates and drugs, has a single base pair polymorphism that creates a premature stop codon that markedly decreases enzyme activity in affected individuals. The ancestral allele (the one that was present in our African forebears) is associated with sodium sensitivity of BP, and as is the case for $A G T$ and GBN3 variants, there is a striking latitudinal gradient for the alleles of CYP $3 A 5$ such that equatorial populations have a high prevalence of the functional allele while northern populations have a high prevalence of the derived, nonfunctional form. In addition, there is a strong correlation of those alleles of CYP $3 A 5$ and AGT that favor sodium sensitivity of BP, suggesting that both responded to the same selective forces created by the migration to northern latitudes.

Adducin is a cytoskeletal protein thought to modulate the activity of sodium-potassium adenosine triphosphatase (ATPase) in renal tubular cells. A polymorphism of the $\alpha$-subunit has been found to be associated with sodium sensitivity of BP and an increased antihypertensive efficacy of hydrochlorothiazide. Here the proposed mechanism is a change in the regulation of the basolateral membrane sodium-potassium ATPase that drives sodium reabsorption throughout the nephron. In the renal proximal tubule, increased action of dopamine suppresses sodium reabsorption, and polymorphisms of several of the genes involved in local dopamine production, receptor activation, and postreceptor signaling have been implicated in sodium-sensitive hypertension. Finally, several reports have observed an association of variants of the $\beta_{2}$-adrenergic receptor and aspects of hypertension, including sodium sensitivity. Other candidate genes that could affect renal sodium handling may affect the

\begin{tabular}{|ll|}
\hline $\begin{array}{l}\text { Table. Genes With Variants Associated With Sodium- } \\
\text { Sensitive Essential Hypertension }\end{array}$ \\
\hline NAME & SyMBOL \\
\hline Angiotensinogen & $A G T$ \\
G-protein $\beta$-3 subunit & $G N B 3$ \\
$\beta_{2}$-Adrenergic receptor & $B 2 A R$ \\
G-protein receptor kinase 4 & $G R K 4$ \\
$\alpha$-Adducin & $A D D$ \\
Cytochrome 3A subtype 5 & $C Y P 3 A 5$ \\
\hline
\end{tabular}

distal tubular epithelial sodium channel. Taken together, these results strongly support the suggestion that genes contributing to differences in renal tubular sodium handling are intimately involved in the predisposition to essential hypertension.

SEARCHING FOR GENES FOR HYPERTENSION The methods used to date to search for genes contributing to hypertension are clearly suboptimal, as they were developed to study phenotypes with many fewer-often single-underlying genes. The first basic approach is genetic linkage, in which various kinds of genomic variations are typed and their genetic transmission from one generation to the next is compared with the transmission of $\mathrm{BP}$ or hypertension in the same individuals. Early applications of this methodology were mostly in large multigenerational families in which individuals were affected by distinctive diseases or conditions. This approach was crucial to the identification of the mutated genes responsible for the rare monogenic forms of hypertension and hypotension; application to essential hypertension was confounded by the problems of genetic heterogeneity previously mentioned as well as by phenotypic heterogeneity when BP itself is used to define the hypertension phenotype.

A related technique, known as linkage dysequilibrium mapping, allow statistical analyses of the relationship of genetic markers to hypertension in nuclear (ie, 2-generation) families or in sibling pairs. The method depends on the phenomenon of meiotic recombination, or crossing over, which creates new combinations of genetic markers during the generation of gametes. The genetic distance between 2 markers can be expressed as a function of how likely it is that a crossover event will occur between them; the likelihood is quantitated as a logarithm of the odds of linkage, the Lod score. By examining how a phenotype such as BP is statistically linked to particular genetic markers, genomic locations harboring genes associated with hypertension can be identified. Linkage dysequilibrium mapping in which markers are typed across the 
entire genome is currently widely used to perform genome scans to identify genomic regions likely to harbor genes contributing to phenotypic variation characterized either as the presence or absence of hypertension or as the quantitative trait of BP. These genomic segments can then be further subdivided by typing more markers in the region, a process known as fine mapping, which can narrow the area of linkage dysequilibrium. When combined with extant genomic maps, such as that created in the Human Genome Project, such approaches may allow identification of a set of specific genes residing in the interval of interest, some of which may contribute to $\mathrm{BP}$ variation.

At this point, linkage approaches merge with the second major approach, that of assessing the association of variants of specific genes with hypertension. Association studies generally compare genetic and phenotypic variation in a case-control design. Because population stratification or genetic admixture can result in spurious associations, careful matching of hypertensive and normotensive subjects for age, sex, and race is important. No matter how carefully matching is performed, however, unknown factors may result in false associations; methods for the detection of stratification and admixture are now routinely applied in association studies to try to prevent such errors. In addition to having relatively straightforward statistics, association studies have the potential appeal of directly identifying causal genetic variants, since allelic differences are directly related to BP differences.

Recent advances in genotyping technology now permit dense saturation of the genome with huge numbers of markers, which should permit wholegenome association studies in the near future. Theoretically, by typing enough genetic variants in multiple individuals with differing phenotypes, it would be possible to examine every gene for an association with BP variation and to identify a set of genes influencing BP regulation. Such an approach would be prohibitively expensive and effort-intensive, but an alternative, termed haplotype mapping, offers the promise of bringing genome-wide association studies into the realm of possibility. This method draws on the fact that our genome is the product of a unique history during which specific individuals had specific meiotic recombination events that were carried forward into their offspring. Between 2 crossover points there is generally a block of DNA unaffected by the events. Within this block, the order of nucleotides is common between any 2 related individuals (except for mutations). By typing a number of individuals from populations with differing population histories (groups that we now perceive as having different geographic origins) a common map of the block structure of the human genome can be created. Once known, those blocks can be characterized by a relatively small set of markers that capture the genetic structure of the nucleotide sequence. These ordered sets of markers are known as haplotypes, and it is the goal of the HapMap Project to develop a set of haplotypes that will span the genome. It may be possible to identify blocks containing genes for hypertension by comparing the distribution of haplotypes between hypertensive and normotensive individuals.

\section{THE FUTURE OF GENETIC STUDIES IN HYPERTENSION}

There is no doubt that within the next few years we will have a wealth of new data regarding specific gene variants that contribute to hypertension. By organizing this information into models that take account of the pathways underlying the physiologic and biochemical mechanisms contributing to BP regulation, we should eventually have the ability to predict who will develop the disease before it appears.

There is considerable interest in strategies directed toward preventing hypertension, and the ability to predict who is at risk will allow us to focus our resources most efficiently. We have had little success in admonishing people to lose weight and to exercise, but it is possible that a better understanding of the evolutionary genetics of hypertension will lead to different approaches to controlling hypertension and the other risk factors of the metabolic syndrome. Perhaps by appreciating how natural selection has preserved systems for rewarding behaviors and preserving metabolic pathways that currently have adverse effects, we will understand how to better prevent the development and progression of risk factors.

Finally, although the subject of much speculation, it remains to be seen whether an understanding of the genetic underpinnings of hypertension will lead to advances in pharmacologic therapy. We understand how genetic polymorphisms in drug metabolizing systems can affect drug levels, but our strategy of dosing antihypertensive drugs (ie, titration of drug dose to an end point of BP level), effectively circumvents the effect of variations in drug metabolism of the commonly used antihypertensives. The hope that insights into pharmacodynamic mechanisms will be aided by understanding 
genetic polymorphisms has not yet shown much promise. Antihypertensive drugs act on targets that are embedded in complex physiologic systems that have the ability to compensate for drug-induced perturbations. Just as single-gene polymorphisms explain relatively little of BP variation, blocking the effects of those alleles may not affect BP much. More importantly, as we understand how those systems are affected by multiple genetic variants, we may be able to identify rational combinations of drugs that block BP regulatory systems at multiple mutually reinforcing functions.

\section{Suggest ed Reading}

- Hunt SC, Gwinn M, Adams TD. Family history assessment: strategies for prevention of cardiovascular disease. Am J Prev Med. 2003;24:136-142.

- Cooper RS. Gene-environment interactions and the etiology of common complex disease. Ann Intern Med. 2003;139:437-440.

- Turner ST, Boerwinkle E. Genetics of blood pressure, hypertensive complications, and antihypertensive drug responses. Pharmacogenomics. 2003;4:53-65.

- O'Shaughnessy KM, Karet FE. Salt handling and hypertension. J Clin Invest. 2004;113:1075-1081.

- Weder AB. Pathogenesis of hypertension: genetic and environmental factors. In: Hollenberg NJ, Braunwald E, eds. Atlas of Hypertension. 5th ed. Philadelphia, PA: Current Medicine, LLC; 2005:1-38.

- Meneton P, Jeunemaitre X, de Wardener HE, et al. Links between dietary salt intake, renal salt handling, blood pressure, and cardiovascular diseases. Physiol Rev. 2005;85:679-715.

- Hunter DJ. Gene-environment interactions in human diseases. Nat Rev Genet. 2005;6:287-298.

- Cordell HJ, Clayton DG. Genetic association studies. Lancet. 2005;366:1121-1131.

- Tanira M, Balushi K. Genetic variations related to hypertension: a review. J Hum Hypertens. 2005;19:7-19.

- Dawn Teare M, Barrett JH. Genetic linkage studies. Lancet. 2005;366:1036-1044.

- Burton PR, Tobin MD, Hopper JL. Key concepts in genetic epidemiology. Lancet. 2005;366:941-951.

- Young JH, Chang YP, Kim JD, et al. Differential susceptibility to hypertension is due to selection during the out-ofAfrica expansion. PLoS Genet. 2005;1:e82.

- Ahn AC, Tewari M, Poon CS, et al. The limits of reductionism in medicine: could systems biology offer an alternative? PLoS Med. 2006;3:e208.

- Ahn AC, Tewari M, Poon CS, et al. The clinical applications of a systems approach. PLoS Med. 2006;3:e209.

- Weder AB. Evolution and hypertension. Hypertension. 2007;49:260-265. 